\title{
CONSTRAINED OUTPUT TRACKING CONTROL FOR TIME-VARYING BILINEAR SYSTEMS VIA RHC WITH INFINITE PREDICTION HORIZON
}

\author{
NGUYEN DOAN PHUOC ${ }^{1}$ AND LE THI THU HA ${ }^{2}$ \\ ${ }^{1}$ Hanoi University of Science and Technology; phuoc.nguyendoan899@gmail.com \\ ${ }^{2}$ Thai Nguyen University of Technology; hahien1977@gmail.com
}

\begin{abstract}
The paper introduces an algorithm to design a feedback controller, which guarantees the tracking of time varying bilinear system outputs for desired values in the presence of input constraint. The proposed controller employs the ideas of receding horizon principle and constrained optimal control. A theorem for the tracking stability of closed loop system is given. An updated law of weighting matrices in the cost function to keep the input constraint condition is also proposed. Finally, the tracking behavior of the closed loop system is illustrated through a numerical example. Keywords. Receding horizon control, constrained nonlinear optimization, dynamic programming, output tracking control.
\end{abstract}

\section{INTRODUCTION}

The problem of output tracking control for nonlinear systems in the presence of constraints is known as an interesting problem of control theory, which has attracted the attention of many control researchers for a long time, but still has not been fully investigated so far. This control problem is attractive since the obtained tracking controller can take into account the limitation of actuators through the input and state constraints, restrict the overshoot of system states as well, and hence prevent damages to system components. Unfortunately, this problem has still not been fully studied due to very large classes of nonlinear systems. Therefore, to effectively solve the problem, a certain class of nonlinear systems as good representative of others should be determined. One of such class is bilinear systems since the bilinear model is the most natural form to express the nonlinearities of industrial processes [1].

There are recently many researches on the control of bilinear systems, however most of them focus only on either the unconstrained tracking performance $[2,3]$, or the constrained stability properties [4-8]. Moreover, to stabilize nonlinear systems with constraints, it is usually recommended to employ MPC techniques in which an appropriate penalty function is added to the cost function. Nevertheless, the question of how to obtain this penalty function for nonlinear MPC is still open.

This paper presents an algorithm to design state feedback tracking controllers for time-varying bilinear systems. This algorithm is constructed based on the conventional receding horizon control (RHC) technique which guarantees the asymptotic tracking of the obtained closed loop system output for a desired value in the presence of input constraints. Especially, the proposed algorithm does not need any additional penalty function in the cost function as introduced in $[7,8]$.

The organization of the paper is as follows. The main results are presented in Section 2 of which a numerical example is also given to illustrate the proposed algorithm. Then some concluding remarks 
are given in Section 3.

\section{MAIN RESULTS}

In this section, the main results on the output tracking controller design for time-varying bilinear systems (also called as controlled subject) modeled by discrete-time equations are presented. The mathematical model of the controlled subject is as follows:

$$
\begin{cases}\underline{x}_{k+1}= & A\left(\underline{x}_{k}, k\right) \underline{x}_{k}+B\left(\underline{x}_{k}, k\right) \underline{u}_{k} \\ \underline{y}_{k}= & C\left(\underline{x}_{k}, k\right) \underline{x}_{k}+D\left(\underline{x}_{k}, k\right) \underline{u}_{k}\end{cases}
$$

where

$$
\begin{aligned}
\underline{x}_{k} & =\left(x_{k}[1], x_{k}[2], \ldots, x_{k}[n]\right)^{T} \in \mathbf{R}^{n} \\
\underline{u}_{k} & =\left(u_{k}[1], u_{k}[2], \ldots, u_{k}[m]\right)^{T} \in \mathbf{R}^{m} \\
\underline{y}_{k} & =\left(y_{k}[1], y_{k}[2], \ldots, y_{k}[m]\right)^{T} \in \mathbf{R}^{m}
\end{aligned}
$$

denote the vectors of system states, inputs and outputs at the time $t_{k}=k T$, respectively and

$$
A\left(\underline{x}_{k}, k\right) \in \mathbf{R}^{n \times n}, B\left(\underline{x}_{k}, k\right) \in \mathbf{R}^{n \times m}, C\left(\underline{x}_{k}, k\right) \in \mathbf{R}^{m \times n}, D\left(\underline{x}_{k}, k\right) \in \mathbf{R}^{m \times m}
$$

are matrices depending on both system states and time.

The tracking control problem for the bilinear system (1) is related to the synthesis of a state feedback controller, which guarantees:

- the asymptotic convergence of output signal $\underline{y}_{k} \rightarrow \underline{y}_{r e f}$, where $\underline{y}_{r e f}$ is a desired reference, and

- the satisfactory of the required input constraints

$$
\underline{u}_{k} \in U
$$

where $U$ is a given subset of control space.

To resolve this tracking control problem, one of the suitable methods is to employ RHC technique.

\subsection{Motivation from conventional receding horizon control}

Recently, RHC which is also referred to as model predictive control (MPC) or moving horizon optimal control (MHOC) is widely admitted to be an effective methodology for solving multivariable constrained control problems. Hitherto, more than 3000 successful applications of RHC have been founded in industry [5].

The main idea of RHC is to minimize a performance index in the form of a certain objective function in the future that would be subjected to constraints on the control signals. Figure 1a) depicts the basic structure of an RHC controller with three main sub-system blocks. The purposes of those three blocks are as follows:

- The first block is predictive model. This block takes the measured system state vector $\underline{x}_{k}$ from the controlled subject (plant) at the current time $t_{k}=k T$ and gives $N$ values of predicted states $\underline{x}_{k+i}, i=1,2, \ldots, N-1$ and predicted outputs $\underline{y}_{k+i}, i=0,1, \ldots, N-1$ in current prediction horizon $[k, N)$. 
Usually, the predictive model is used with the same discrete-time equations (1) of plant. Therefore, the predictive output vector $\underline{y}_{k+i}$ is obtained from this predictive model which in general is expressed as a function of future inputs $\underline{u}_{k+i}, i=0,1, \ldots, N-1$ as follows:

$$
\begin{aligned}
& \underline{x}_{k+i}=\underline{p}_{i}(\mathcal{U}), i=1,2, \ldots, N-1 \\
& \underline{y}_{k+i}=\underline{q}_{i}(\mathcal{U}), i=0,1, \ldots, N-1
\end{aligned}
$$

where the vector $\mathcal{U}$ is defined as:

$$
\mathcal{U}=\operatorname{col}\left(\underline{u}_{k}, \underline{u}_{k+1}, \ldots, \underline{u}_{k+N-1}\right) \in \mathbf{R}^{m N}
$$

- The second block is any chosen objective function $J$ according to the desired performance of closed loop system. The following objective functions could be employed:

$$
J=\sum_{i=0}^{N-1}\left(\underline{x}_{k+i}^{T} Q \underline{x}_{k+i}+\underline{u}_{k+i}^{T} R \underline{u}_{k+i}\right)
$$

for the stability of closed loop system, or

$$
J=\sum_{i=0}^{N-1}\left(\underline{e}_{k+i}^{T} Q \underline{e}_{k+i}+\underline{u}_{k+i}^{T} R \underline{u}_{k+i}\right)
$$

for the output tracking to a desired output vector, where $\underline{e}_{k+i}=\underline{y}_{r e f}-\underline{y}_{k+i}$, are tracking errors in the current prediction horizon $[k, N)$ and are any symmetric positive definite matrices. Together with $(3)$ it is obviously that the objective function $J(\mathcal{U})$ at current time $t_{k}=k T$ is a function which only depends on the vector $\mathcal{U}$.

- The last block is an optimization algorithm applied to solve optimization problem:

$$
\mathcal{U}^{*}=\arg \min _{\mathcal{U} \in U^{N}} J(\mathcal{U})
$$

subjected to the input constraint $U^{N} \subset \mathbf{R}^{m N}$, where $J(\mathcal{U})$ is obtained from the second block. Generally, (7) is a nonlinear optimization problem of which the objective function $J(\mathcal{U})$ is not a quadratic function of $\mathcal{U}$. Hence, sequential quadratic programming (SQP) is one of the most used algorithm in the implementation of (7), which is known as a successful method to solve a constrained nonlinear optimization problem off-line [9].

Finally, only the first element $\underline{u}_{k}^{*}$ of resulting optimal sequence $\mathcal{U}^{*}=\operatorname{col}\left(\underline{u}_{k}^{*}, \underline{u}_{k+1}^{*}, \ldots, \underline{u}_{k+N-1}^{*}\right)$ is sent to the plant as the control signal during the time interval $k T \leq t<(k+1) T$ whereas the others are discarded. At the next time instant $t_{k+1}=(k+1) T, k=0,1, \ldots$ all calculating steps above are repeated to find the new control signal $\underline{u}_{k+1}^{*}$ with the prediction horizon moved forward as described in Figure 1b). In that way, RHC is a type of quasi-optimal control, which has the feature that constraints can be implemented in the controller. This helps the system operating efficiently and preventing equipments from damages.

On the other hand, conventional RHC has three main disadvantages: 
1. While RHC requires the iterative off-line solution of nonlinear optimization problem (7) on a finite prediction horizon, which is generally not convex, the obtained $\mathcal{U}^{*}$ may not be the global solution. And if $\mathcal{U}^{*}$ is only a local solution, the control performance would be bad.

2. The finiteness of the prediction horizon impacts also badly on the performance of closed loop system. If the prediction horizon is not chosen large enough, the closed loop system would be unstable, especially for nonlinear systems.

3. Furthermore, conventional RHC controllers usually need a huge computational power due to the use of a nonlinear optimization algorithm such as SQP to solve the nonlinear optimization problem (7).

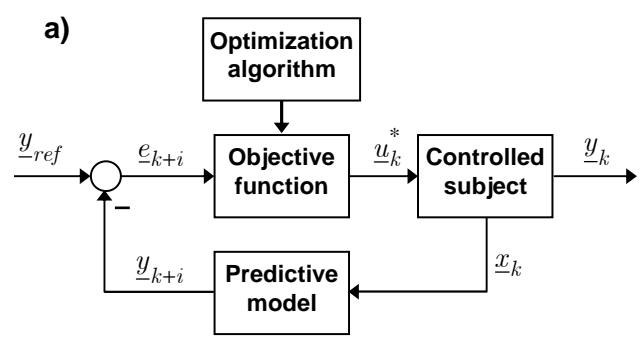

b)

Figure 1: Basic structure of an RHC controller

So ideally, instead of using finite prediction horizon $[k, N)$ and applying SQP or other similar nonlinear optimization algorithms to obtain an off-line solution $\underline{u}_{k}$ of $(7)$, an infinite horizon $[k, \infty]$ would be utilized and an optimal control method such as the variation technique or the dynamic programming would be implemented to determine an on-line solution $\underline{u}_{k}\left(\underline{x}_{k}\right)$ associated with a timeinvariant cost function over the infinite horizon :

$$
J=\sum_{i=0}^{\infty}\left(\underline{x}_{k+i}^{T} Q \underline{x}_{k+i}+\underline{u}_{k+i}^{T} R \underline{u}_{k+i}\right) \rightarrow \min
$$

This satisfies the required constraint $\underline{u}_{k+i} \in U$. However, a solution of such constrained optimal problem (8) with constant weighting matrices $Q, R$ cannot be analytically found in general. Thus, this paper presents an approach to overcome the mentioned problems for time-varying bilinear system (1). This approach is based on the repeating solution of optimal control problem with an infinite time-varying cost function:

$$
J_{k}=\sum_{i=0}^{\infty}\left(\underline{x}_{k+i}^{T} Q_{k} \underline{x}_{k+i}+\underline{u}_{k+i}^{T} R_{k} \underline{u}_{k+i}\right) \rightarrow \min
$$

which is moved forward together with the prediction horizon $[k, \infty]$, where their time-dependent weighting matrices $Q_{k}, R_{k}$ are updated to correspond with the required constraint of $\underline{u}_{k}$ given in (2) after each moving step. 


\subsection{Receding state feedback control with infinite horizon}

Since the on-line optimal state feedback controller $\underline{u}_{k}\left(\underline{x}_{k}\right)$, which is directly obtained via the dynamic programming technique, can only be applied for the stabilizing problem, not for the tracking problem, the aforementioned constrained tracking control problem for the time-varying bilinear system (1) should be converted to a stabilizing control problem. While the vector $\underline{x}_{k}$ of system states at the current time instant $t_{k}=k T$ is assumed to be measurable, the given time-varying bilinear system (1) can be considered as a linear time-varying system during the time interval $k T \leq t<(k+1) T$, as follows:

$$
\left\{\begin{array}{l}
\underline{x}_{k+1}=\mathcal{A}_{k} \underline{x}_{k}+\mathcal{B}_{k} \underline{u}_{k} \\
\underline{y}_{k}=\mathcal{C}_{k} \underline{x}_{k}+\mathcal{D}_{k} \underline{u}_{k}
\end{array}\right.
$$

where $\mathcal{A}_{k}=A\left(\underline{x}_{k}, k\right), \mathcal{B}_{k}=B\left(\underline{x}_{k}, k\right), \mathcal{C}_{k}=C\left(\underline{x}_{k}, k\right), \mathcal{D}_{k}=D\left(\underline{x}_{k}, k\right)$ are all determined matrices at the current time $t_{k}$. Moreover, if the state vector and control signals of (10) at the tracking steady state are denoted by $\underline{x}_{s}, \underline{u}_{s}$, then these values must satisfy:

$$
\begin{gathered}
\left\{\begin{array}{l}
\underline{x}_{s}=\mathcal{A}_{k} \underline{x}_{s}+\mathcal{B}_{k} \underline{u}_{s} \\
\underline{y}_{r e f}=\mathcal{C}_{k} \underline{x}_{s}+\mathcal{D}_{k} \underline{u}_{s}
\end{array}\right. \\
\Leftrightarrow\left(\begin{array}{cc}
\mathcal{A}_{k}-I_{n} & \mathcal{B}_{k} \\
\mathcal{C}_{k} & \mathcal{D}_{k}
\end{array}\right)\left(\begin{array}{c}
\underline{x}_{s} \\
\underline{u}_{s}
\end{array}\right)=\left(\begin{array}{c}
\underline{0}_{\underline{y}_{r e f}}
\end{array}\right)
\end{gathered}
$$

where $I_{n}$ is the $n \times n$ identity matrix. Therefore, if the following assumption is true:

Assumption 1. The matrix:

$$
G_{k}=\left(\begin{array}{cc}
\mathcal{A}_{k}-I_{n} & \mathcal{B}_{k} \\
\mathcal{C}_{k} & \mathcal{D}_{k}
\end{array}\right) \in \mathbf{R}^{(n+m) \times(n+m)}
$$

is invertible for all $k$.

then both steady state vectors $\underline{x}_{s}, \underline{u}_{s}$ of the system (10) are uniquely obtained from:

$$
\left(\begin{array}{l}
\underline{x}_{s} \\
\underline{u}_{s}
\end{array}\right)=G_{k}^{-1}\left(\begin{array}{c}
\underline{0} \\
\underline{y}_{r e f}
\end{array}\right)=\left(\begin{array}{cc}
\mathcal{A}_{k}-I_{n} & \mathcal{B}_{k} \\
\mathcal{C}_{k} & \mathcal{D}_{k}
\end{array}\right)^{-1}\left(\begin{array}{c}
\underline{0} \\
\underline{y}_{r e f}
\end{array}\right)
$$

Now, define the deviated values from steady state as follows:

$$
\begin{aligned}
\underline{\delta}_{k} & =\underline{x}_{k}-\underline{x}_{s} \\
\underline{\rho}_{k} & =\underline{u}_{k}-\underline{u}_{s}
\end{aligned}
$$

then the original tracking control problem of system (10) can be appropriately converted to the stabilizing problem of the following system, which is obtained by subtracting (10) and (11):

$$
\underline{\delta}_{k+1}=\mathcal{A}_{k} \underline{\delta}_{k}+\mathcal{B}_{k} \underline{\rho}_{k}
$$

in the presence of input constraint:

$$
\underline{\rho}_{k} \in \Delta \text { with } \Delta=\left\{\underline{\rho} \in \mathbf{R}^{m} \mid \underline{\rho}+\underline{u}_{s} \in U\right\}
$$


This now can be seen as the nominal system of (10). Subsequently, to optimally stabilize the nominal system $(15)$ over the infinite prediction horizon $[k, \infty]$, the following cost functions is used:

$$
J_{k}=\sum_{i=k}^{\infty}\left(\underline{\delta}_{i}^{T} Q_{k} \underline{\delta}_{i}+\underline{\rho}_{i}^{T} R_{k} \underline{\rho}_{i}\right) \rightarrow \min
$$

Where two symmetric positive definite weighting matrices $Q_{k}, R_{k}$ will be updated by each moving step of prediction horizon along time axis while taking into account the input constraint (16). In the unconstrained scenario and based on dynamical programming, the on-line solution $\underline{\delta}_{k}\left(\underline{\rho}_{k}\right)$ of formulated optimal control problem (17) for nominal system (15) is already given in [9] as follows:

$$
\underline{\rho}_{k}=-\left(R_{k}+\mathcal{B}_{k}^{T} L_{k} \mathcal{B}_{k}\right)^{-1} \mathcal{B}_{k}^{T} L_{k} \mathcal{A}_{k} \underline{\delta}_{k}
$$

where the matrix $L_{k}$ is obtained from following discrete Riccati equation:

$$
L_{k}=Q_{k}+\mathcal{A}_{k}^{T} L_{k} \mathcal{A}_{k}-\mathcal{A}_{k}^{T} L_{k} \mathcal{B}_{k}\left(R_{k}+\mathcal{B}_{k}^{T} L_{k} \mathcal{B}_{k}\right)^{-1} \mathcal{B}_{k}^{T} L_{k} \mathcal{A}_{k}
$$

It can be easily seen from equations (18) and (19), that the module of $\underline{\rho}_{k}$ depends on two weighting matrices $Q_{k}=Q_{k}^{T}>0$ and $R_{k}=R_{k}^{T}>0$, which are arbitrarily selected. More precisely, the bigger rate $\left\|R_{k}\right\| /\left\|Q_{k}\right\|$ is, the smaller $\left\|\underline{\rho}_{k}\right\|$ will be. Hence, in constrained circumstance, $Q_{k}, R_{k}$ can also be appropriately chosen, such is together with the forward movement of infinite prediction horizon $[k, \infty], k=0,1, \ldots$ along the time axis, then, the obtained $\underline{\rho}_{k}$ will satisfy the required input constraint given in (16).

However, some conditions of the stabilizing problem may arise in application, where there is no solution $\underline{\rho}_{k}$ to the corresponding optimal control problem (17) that satisfies the constraint (16). Thereby, for the feasibility of original stabilizing control problem, the following assumption is needed.

Assumption 2. At each sampling time $k T, k=0,1, \ldots$ there always exist two symmetric positive definite matrices $Q_{k}, R_{k}$ such that $\underline{\rho}_{k}$ is obtained from (18) and (19) satisfies (16).

Finally, the constrained tracking control signal $\underline{u}_{k}$ for the original time-varying bilinear system (1) can be now recovered from the obtained optimal solution $\underline{\rho}_{k}$ associated with equations (18), (19) as follows:

$$
\underline{u}_{k}=\underline{\rho}_{k}+\underline{u}_{s}
$$

The following algorithm summarizes all iterative calculating steps above to determine the expected tracking control signal $\underline{u}_{k}$ for time-varying bilinear system (1), which satisfies the constraint (2).

Algorithm 1. Constrained tracking control of time-varying bilinear systems

1. Select any two symmetric positive definite matrices $Q$ and $R$. Specify two updated factors $0<\eta<1,1<\mu$. Set $k=0$.

2. Measure the current state vector $\underline{x}_{k}$ from system (1). Calculate $\mathcal{A}_{k}=A\left(\underline{x}_{k}, k\right), \mathcal{B}_{k}=$ $B\left(\underline{x}_{k}, k\right), \mathcal{C}_{k}=C\left(\underline{x}_{k}, k\right), \mathcal{D}_{k}=D\left(\underline{x}_{k}, k\right)$ and determine $\underline{x}_{s}, \underline{u}_{s}$ of (1) according to (13).

3. Calculate $\underline{\rho}$ as follows: $\underline{\rho}=-\left(R+\mathcal{B}_{k}^{T} L \mathcal{B}_{k}\right)^{-1} \mathcal{B}_{k}^{T} L \mathcal{A}_{k}\left(\underline{x}_{k}-\underline{x}_{s}\right)$ where the matrix is obtained from: $L=Q+\mathcal{A}_{k}^{T} L \mathcal{A}_{k}-\mathcal{A}_{k}^{T} L \mathcal{B}_{k}\left(R+\mathcal{B}_{k}^{T} L \mathcal{B}_{k}\right)^{-1} \mathcal{B}_{k}^{T} L \mathcal{A}_{k}$

4. If $\underline{\rho} \notin \Delta$ with $\Delta$ is given in (16), then set $R:=\mu R$ and go back to the step 3 . Otherwise go to the next step. 
5. Send $\rho+\underline{u}_{s}$ to the system (1) during the time interval $k T \leqslant t<(k+1) T$ as the control signal $\underline{u}_{k}$. Then set $R:=\eta R, k:=k+1$ and go back to the step 2 .

Figure 2 depicts the obtained closed loop system using proposed algorithm as an infinite horizon state feedback RHC controller.

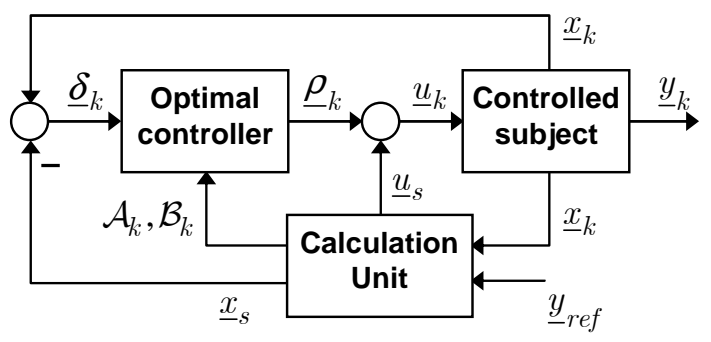

Figure 2: Output tracking RHC controller

\subsection{Tracking stability of the closed loop system}

To verify the output tracking behavior of closed loop system depicted in Figure 2 in the sense, that all system signals $\underline{y}_{k}, \underline{x}_{k}, \underline{u}_{k}$ asymptotically converge on desired values $\underline{y}_{r e f}, \underline{x}_{s}, \underline{u}_{s}$ respectively, two aforementioned assumptions are needed. It can be seen that these assumptions make the desired performance:

$$
\underline{y}_{k} \rightarrow \underline{y}_{r e f}, \underline{x}_{k} \rightarrow \underline{x}_{s}, \underline{u}_{k} \rightarrow \underline{u}_{s}
$$

satisfied if and only if the nominal system (15) is asymptotically stable. And now, this expected asymptotic stability of the nominal system (15) is obtained from the following theorem.

Theorem 1. The time-varying closed loop system involving the nominal system (15) and the state feedback optimal controller (18) is asymptotically stable.

Proof. Denote the vector of control signals of the closed loop system by (Figure 3a):

$$
\underline{\rho}(t)=\underline{\rho}_{k} \text { for } k T \leq t<(k+1) T
$$

then the vector of system states can be described during the time interval by:

$$
\underline{\delta}_{k+1}=\mathcal{A}_{k} \underline{\delta}_{k}+\mathcal{B}_{k} \underline{\rho}_{k}=\left[\mathcal{A}_{k}-\mathcal{B}_{k}\left(R_{k}+\mathcal{B}_{k}^{T} L_{k} \mathcal{B}_{k}\right)^{-1} \mathcal{B}_{k}^{T} L_{k} \mathcal{A}_{k}\right] \underline{\delta}_{k}=F_{k} \underline{\delta}_{k}
$$

where

$$
F_{k}=\mathcal{A}_{k}-\mathcal{B}_{k}\left(R_{k}+\mathcal{B}_{k}^{T} L_{k} \mathcal{B}_{k}\right)^{-1} \mathcal{B}_{k}^{T} L_{k} \mathcal{A}_{k}
$$

Hence it follows that the state vector of the closed loop system in the whole control time $t \geq 0$ is:

$$
\begin{aligned}
\underline{\delta}_{k} & =F_{k-1} \underline{\delta}_{k-1}=F_{k-1} F_{k-2} \underline{\delta}_{k-2}=\left(F_{k-1} F_{k-2} \cdots F_{k-i}\right) \underline{\delta}_{k-i} \\
& \vdots \\
& =\prod_{i=1}^{k} F_{k-i} \underline{\delta}_{0}=\mathcal{F}_{k} \underline{\delta}_{0}
\end{aligned}
$$


where $\mathcal{F}_{k}$ is the matrix defined as:

$$
\mathcal{F}_{k}=\prod_{i=1}^{k} F_{k-i}
$$

Furthermore, due to the positive definiteness of $Q_{k}, R_{k}$ it is obtained:

$$
\begin{aligned}
J_{k}^{*} & =\min \sum_{i=k}^{\infty}\left(\underline{\delta}_{i}^{T} Q_{k} \underline{\delta}_{i}+\underline{\rho}_{i}^{T} R_{k} \underline{\rho}_{i}\right)<\infty \\
& \Rightarrow\left(\underline{\delta}_{i}^{T} Q_{k} \underline{\delta}_{i}+\underline{\rho}_{i}^{T} R_{k} \underline{\rho}_{i}\right) \rightarrow 0 \\
& \Rightarrow \underline{\delta}_{i} \rightarrow \underline{0} \text { and } \underline{\rho}_{i} \rightarrow \underline{0}
\end{aligned}
$$

Therefore, all matrices $F_{k}, k=1,2, \ldots$ given in (22) are Schur matrices [9]. As a result, $\mathcal{F}_{k}$ defined in (23) is also a Schur matrix and satisfies: $\lim _{k \rightarrow \infty} \mathcal{F}_{k}=\Theta$ From this point and together with equation (18), it can be easily point out that (Figure $3 \mathrm{~b}$ ): $\lim _{k \rightarrow \infty} \underline{\rho}_{k}=\underline{0}$ and this is equivalent to the asymptotic stability subject to the input constraint (16) of the nominal system (15), which is controlled by $\underline{\rho}(t)$ given in (21). Hence, the tracking stability of closed loop system depicted in Figure 2 (in the sense of (20)) subject to input constraint (2) can be deduced from Assumptions 1 and 2 .

a)

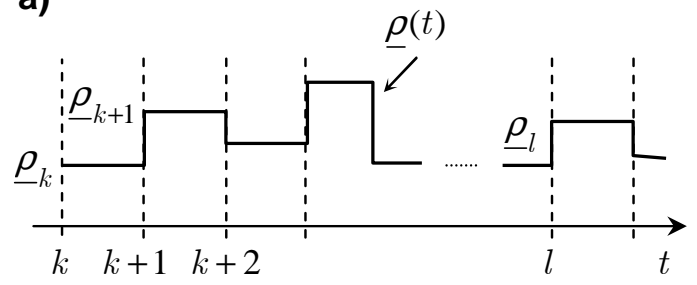

b)

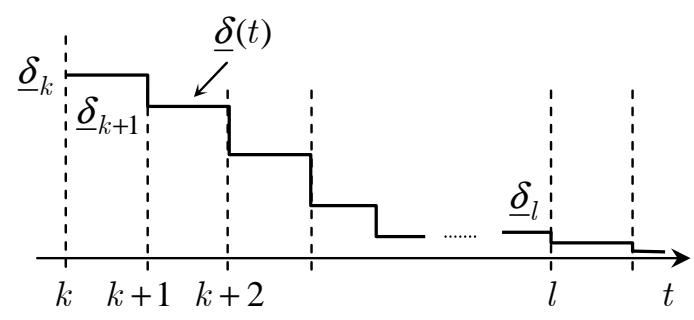

Figure 3: To proof the tracking stability of closed loop system

\subsection{Numerical example}

Hereafter, the proposed algorithm will be illustrated through the tracking control problem for following second order time-varying bilinear system:

$$
\left\{\begin{array}{l}
\underline{x}_{k+1}=\left(\begin{array}{cc}
1 & x_{k}^{2}[1] \\
1 & 0.5
\end{array}\right) \underline{x}_{k}+\left(\begin{array}{c}
1 \\
1
\end{array}\right) u_{k} \\
y_{k}=(1,1) \underline{x}_{k}
\end{array}\right.
$$

with the input constraint $\left|u_{k}\right| \leqslant 1$, where $\underline{x}_{k}=\left(x_{k}[1], x_{k}[2]\right)^{T}$ denotes the state vector. This system has the matrix $G_{k}$ defined in (12):

$$
G_{k}=\left(\begin{array}{cc}
\mathcal{A}_{k}-I_{n} & \mathcal{B}_{k} \\
\mathcal{C}_{k} & \mathcal{D}_{k}
\end{array}\right)=\left(\begin{array}{ccc}
0 & x_{k}^{2}[1] & 1 \\
1 & -0.5 & 1 \\
1 & 1 & 0
\end{array}\right)
$$


which is invertible for all $k$, because of $\operatorname{det}\left(G_{k}\right)=x_{k}^{2}[1]+1.5 \neq 0$ for all $\underline{x}_{k}$. Hence, Assumption 1 is satisfied. Furthermore, with desired value $y_{r e f}$, the system state vector $\underline{x}_{s}$ and the control input $u_{s}$ at the steady state are obtained according to equation (13) as follows:

$$
\begin{aligned}
\left(\begin{array}{l}
\underline{x}_{s} \\
u_{s}
\end{array}\right) & =\left(\begin{array}{ccc}
0 & x_{k}^{2}[1] & 1 \\
1 & -0.5 & 1 \\
1 & 1 & 0
\end{array}\right)^{-1}\left(\begin{array}{c}
0 \\
0 \\
y_{\text {ref }}
\end{array}\right)=\frac{y_{\text {ref }}}{x_{k}^{2}[1]+1.5}\left(\begin{array}{c}
x_{k}^{2}[1]+0.5 \\
1 \\
x_{k}^{2}[1]
\end{array}\right) \\
& \Rightarrow 0 \leq\left|u_{s}\right| \leq\left|y_{\text {ref }}\right|
\end{aligned}
$$

Therefore, in the case of $\left|y_{r e f}\right|<1$ the control problem could be feasible, or Assumption 2 could be satisfied.

Figure 4a) exhibits the control signal $u_{k}$ and Figure $4 \mathrm{~b}$ ) displays the real output signal $y_{k}$ of closed loop system, which is obtained by simulation with: $Q=10 I_{2}, R=1, \mu=2$ and $y_{\text {ref }}=0.5$ for two separated updated cases $\eta_{1}=1 / 4$ (dashed line) and $\eta_{2}=1 / 3$ (dot line). They show that the output $y_{k}$ for both cases converges asymptotically on the desired value $y_{r e f}$, while $u_{k}$ still belongs to the subset $\left|u_{k}\right| \leq 1$ of control space.

a)

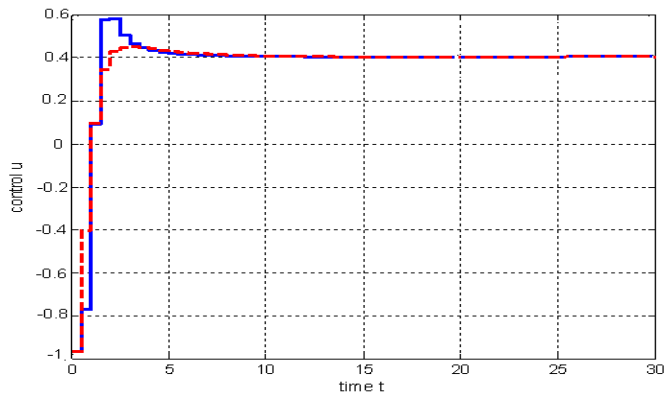

b)

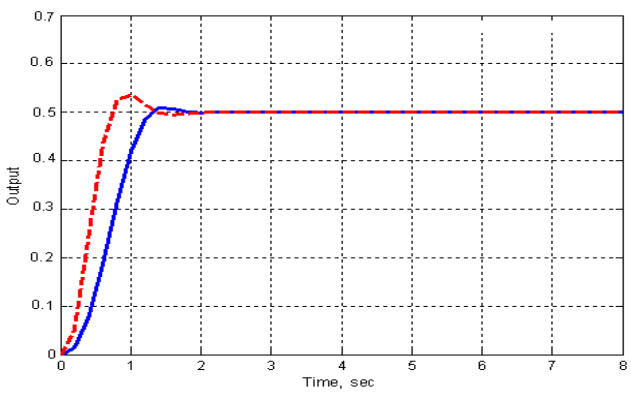

Figure 4: Simulation results

\section{CONCLUSIONS}

The output tracking control problem of time-varying bilinear systems subject to input constraints is under study in this paper. A framework is proposed to design the state feedback controller, which guarantees both the output tracking of the closed loop system subject to given input constraints and the asymptotic stability of corresponding nominal system. The main idea of the proposed design framework is based on RHC strategy over the infinite prediction horizon and an updated law of weighting matrices $Q_{k}, R_{k}$ in the cost function moving forward together with the infinite prediction horizon $[k, \infty], k=0,1, \ldots$ along the time axis. The key for this parameter updating is the fact that the bigger rate $\left\|R_{k}\right\| /\left\|Q_{k}\right\|$ is, the smaller variation values $\left\|\rho_{k}\right\|$ of the nominal system input will be. A numerical example is presented to illustrate the applicability of the proposed constrained output tracking controller into practice.

Finally, whereas the proposed framework is primary constructed for discrete time system (1), it 
can be also applied to control continuous time bilinear systems described by:

$$
\left\{\begin{array}{l}
\underline{\dot{x}}=A(\underline{x}, t) \underline{x}+B(\underline{x}, t) \underline{u} \\
y=C(\underline{x}, t) \underline{x}+D(\underline{x}, t) \underline{u}
\end{array}\right.
$$

if there are all matrices in it continuous, because by using the following approximation for the differentiation:

$$
\underline{\dot{x}} \approx \frac{\underline{x}_{k+1}-\underline{x}_{k}}{T}
$$

in a sufficiently small sampling time, the continuous time system (24) will be converted suitably to:

$$
\left\{\begin{array}{l}
\underline{x}_{k+1}=\left[I+T A\left(\underline{x}_{k}, k\right)\right] \underline{x}_{k}+T B\left(\underline{x}_{k}, k\right) \underline{u}_{k} \\
\underline{y}_{k}=C\left(\underline{x}_{k}, k\right) \underline{x}_{k}+D\left(\underline{x}_{k}, k\right) \underline{u}_{k}
\end{array}\right.
$$

This is obviously equivalent to (1).

\section{REFERENCES}

[1] P. M. Pardalos and V. A. Yatsenko, Optimization and Control of Bilinear Systems: Theory, Algorithms, and Applications. Springer Science \& Business Media, 2008, vol. 11.

[2] V. Y. Belozyorov, "Design of linear feedbacks for bilinear control systems," International Journal of Applied Mathematics and Computer Science, vol. 12, no. 4, pp. 493-512, 2002.

[3] H. Ramezanpour, S. Setayeshi, H. Arabalibeik, and A. Jajrami, "An iterative procedure for optimal control of bilinear systems," International Journal of Instrumentation and Control Systems, vol. 2 , no. 1, pp. 1-10, 2012.

[4] M. Bacic, M. Cannon, and B. Kouvaritakis, "Constrained control of siso bilinear systems," $A u-$ tomatic Control, IEEE Transactions on, vol. 48, no. 8, pp. 1443-1447, 2003.

[5] R. Findeisen, L. Imsland, F. Allgöwer, and B. A. Foss, "State and output feedback nonlinear model predictive control: An overview," European Journal of Control, vol. 9, no. 2-3, pp. 190-206, 2003.

[6] A. B. Fontes, C. E. Dórea, and M. R. d. S. Garcia, "An iterative algorithm for constrained mpc with stability of bilinear systems," in 16th Mediterranean Conference on Control and Automation. IEEE, 2008, pp. 1526-1531.

[7] L. Grüne and J. Pannek, Nonlinear model predictive control. Springer, 2011.

[8] J. M. Maciejowski, Predictive control: with constraints. Pearson education, 2002.

[9] N. D. Phuoc, Toi uu hoa trong dieu khien va dieu khien toi uu. Bach Khoa Publishing House, (To be published 2015, in Vietnamese).

Received on January 28, 2015

Revised on June 12, 2015 